\title{
Synchronized reinjection and coalescence of droplets in microfluidics
}

\section{Citation}

Lee, Manhee, Jesse W. Collins, Donald M. Aubrecht, Ralph A. Sperling, Laura Solomon, JongWook Ha, Gi-Ra Yi, David A. Weitz, and Vinothan N. Manoharan. 2014. "Synchronized Reinjection and Coalescence of Droplets in Microfluidics." Lab Chip 14 (3): 509-513. doi:10.1039/c3lc51214b.

\section{Published Version}

doi:10.1039/c3lc51214b

\section{Permanent link}

http://nrs.harvard.edu/urn-3:HUL.InstRepos:24902771

\section{Terms of Use}

This article was downloaded from Harvard University's DASH repository, and is made available under the terms and conditions applicable to Open Access Policy Articles, as set forth at http:// nrs.harvard.edu/urn-3:HUL.InstRepos:dash.current.terms-of-use\#OAP

\section{Share Your Story}

The Harvard community has made this article openly available.

Please share how this access benefits you. Submit a story.

\section{Accessibility}




\title{
Synchronized reinjection and coalescence of droplets in microfluidics ${ }^{\dagger}$
}

\author{
Manhee Lee, ${ }^{a, \S}$ Jesse W. Collins, ${ }^{b}$ Donald M. Aubrecht, ${ }^{b}$ Ralph A. Sperling, ${ }^{b, \#}$ Laura Solomon, ${ }^{c}$ Jong- \\ wook Ha, ${ }^{d}$ Gi-Ra Yi, ${ }^{e}$ David A. Weitz, ${ }^{a, b}$ and Vinothan N. Manoharan ${ }^{* b, a}$ \\ Received (in XXX, XXX) Xth XXXXXXXXX 20XX, Accepted Xth XXXXXXXXX 20XX \\ ${ }_{5}$ DOI: $10.1039 / \mathbf{b 0 0 0 0 0 0 x}$
}

Coalescence of two kinds of pre-processed droplets is necessary to perform chemical and biological assays in droplet-based microfluidics. However, a robust technique to accomplish this does not exist. Here we present a microfluidic device to synchronize the reinjection of two different kinds of droplets and coalesce them, using hydrostatic pressure in conjunction with a conventional syringe pump. We use a 10 device consisting of two opposing T-junctions for reinjecting two kinds of droplets and control the flows of the droplets by applying gravity-driven hydrostatic pressure. The hydrostatic-pressure operation facilitates balancing the droplet reinjection rates and allows us to synchronize the reinjection. Furthermore, we present a simple but robust module to coalesce two droplets that sequentially come into the module, regardless of their arrival times. These re-injection and coalescence techniques might be used

15 in lab-on-chip applications requiring droplets with controlled numbers of solid materials, which can be made by coalescing two pre-processed droplets that are formed and sorted in devices.

\section{Introduction}

In droplet-based microfluidics, droplets are used as "microreactors" for chemical and biological assays. ${ }^{1}$ The precise 20 addition of reagents into the droplets is essential for this function. Methods such as "picoinjection," where reagents contained in a pressurized channel are forced into re-injected target droplets under a controlled electric field, ${ }^{2}$ are primarily useful for adding liquid reagents. It is more difficult to control the addition of small 25 solid particles using such methods because of Poisson fluctuations in the particle number. ${ }^{3}$ The most general way to combine both solid and liquid materials such as beads, cells, and solutions is to coalesce pairs of previously prepared target and reagent droplets.

30 However, existing methods for coalescing droplets ${ }^{4-8}$ generally work only for droplets that are formed on-chip. Because they require the droplet streams to be ordered prior to coalescence, they are not well-suited to coalescing droplets that are formed, sorted, incubated, or prepared in external devices. Moreover, 35 existing coalescers require specific conditions such as uniform droplet-droplet distances ${ }^{7}$ or constant flow rates, ${ }^{9,10}$ so that the user must first match the initial flow rate of the droplets to the operating conditions of the coalescer.

Here we introduce a simple but robust microfluidic device that 40 overcomes these limitations, allowing pre-prepared droplets to be reinjected and coalesced in high yield under a wide range of flow conditions. The key enabling feature is the use of gravity-driven hydrostatic pressure, rather than syringe-pump driven flow, to drive the droplets through the device. We show that the device 45 can produce a stream of alternating droplets of two different types and coalesce them with $85 \%$ yield or higher. Furthermore, because the coalescer operates over a wide range of flow conditions, it can be easily incorporated into practical microfluidic applications.
Our microfluidic devices are fabricated using standard softlithography in poly-dimethylsiloxane (PDMS). ${ }^{11}$ They consist of PDMS channels bonded to a glass slide and have rectangular cross-sections with a constant height of $25 \mu \mathrm{m}$ (Fig. 1(a)), which ${ }_{55}$ is determined by the thickness of the photoresist used (SU8-3025) and is controlled by the rotation frequency of a spin-coater. The channels are treated with a surface coating agent (Aquapel, PPG Industries) to make them fluorophilic and are subsequently flushed with nitrogen gas.

60 The droplet emulsions are made with microfluidic flowfocusing devices, as shown in Fig. 1(a). For the dispersed phase we use deionized water, to which we add dye (Allura Red, SigmaAldrich) for visualizing the droplets. The continuous phase is fluorinated oil (HFE-7500, 3M) containing $1-3 \% \mathrm{w} / \mathrm{w}$ ${ }_{65}$ surfactant. We synthesize and use two different surfactants, surfactant I and surfactant II (see ESI $1 \uparrow$ for details). Most experiments are conducted using surfactant I, except the experiments with device III in Figs. 3(a,c), where we use surfactant II for emulsion production and reinjection. Each of the 70 phases is injected into the devices at a constant flow rate by a syringe pump, resulting in highly monodisperse droplets that emerge at a rate of about 1000 per second. Figure 1(b) shows typically formed droplets and their size distribution.

The emulsions formed on-chip are delivered to and stored in 75 storage chambers that are connected to reservoirs containing the same surfactant-laden oil used in emulsion production (Fig. 1(a)). The storage chambers and reservoirs allow us to use hydrostatic pressure to reinject droplets into another device. The pressure is controlled by varying the heights of the reservoirs. The emulsions 80 are stored for 1-2 days before they are fed to the reinjection and coalescence devices. We do this to ensure that our devices work with pre-processed droplets, which in other experiments might be stored for some time before further processing. In our experiments we always use the same carrier oil containing the ${ }_{85}$ same surfactant as that used for emulsion production.

\section{${ }_{50}$ Materials and methods}



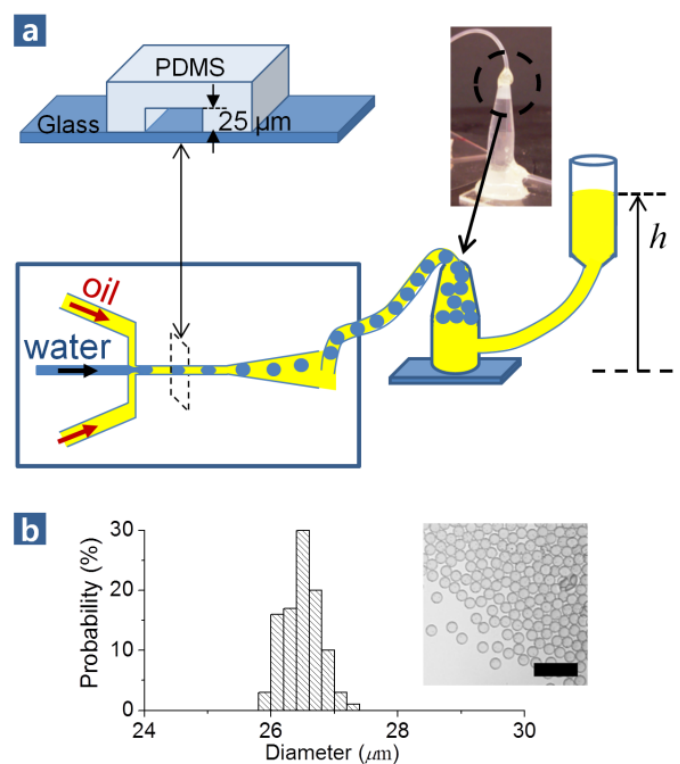

Fig. 1. Droplet production for re-injection and coalescence. (a) Microfluidic devices are made by standard soft-lithography in PDMS. All have rectangular cross-sections and a constant height $h=25 \mu \mathrm{m}$. To 5 produce emulsions for reinjection and coalescence, we use flow-focusing devices with fluorinated oil (HFE-7500) containing 1-3\% w/w surfactant. The droplets are delivered into storage chambers connected to reservoirs. By varying the height of the reservoir, we control the outlet pressure of the device, which facilitates collecting, storing, and reinjecting droplets.

10 (b) Droplets formed in the flow-focusing device are highly monodisperse. Here we selected 100 droplets and measured their diameters using image analysis. The inset shows an optical micrograph of droplets that have just formed and have reached the collection outlet. The scale bar is $100 \mu \mathrm{m}$.

\section{Results and discussions}

15 Pressure-driven operation provides two advantages for coalescing two different kinds of droplets. First, because the pressure drop in our device, shown in Fig. 2(a), can be controlled with a resolution of about $100 \mathrm{~Pa}$, droplets can be reinjected one at a time, and the injection frequency can be varied with $1 \mathrm{~Hz}$ resolution. Second, 20 the pressure in the device stabilizes within a few seconds, allowing the user to stabilize the droplets' relative flow rates at least an order of magnitude faster than in devices driven by syringe pumps, which operate under a constant flow-rate condition and suffer long-lasting pressure transients. ${ }^{12}$

25

We first demonstrate reinjecting a single type of droplet. As shown in Fig. 2(a), we drive the flow of the continuous oil phase at constant flow rate $Q_{\mathrm{m}}$ using a syringe pump, and we drive the reinjection of droplets from the storage chamber into the device 30 using a constant hydrostatic pressure $P_{\text {io }}$, which is determined by the height difference between oil reservoirs at inlet and outlet, $P_{\mathrm{io}} \equiv P_{\mathrm{i}}-P_{\mathrm{o}} \propto\left(h_{\mathrm{i}}-h_{\mathrm{o}}\right)$.

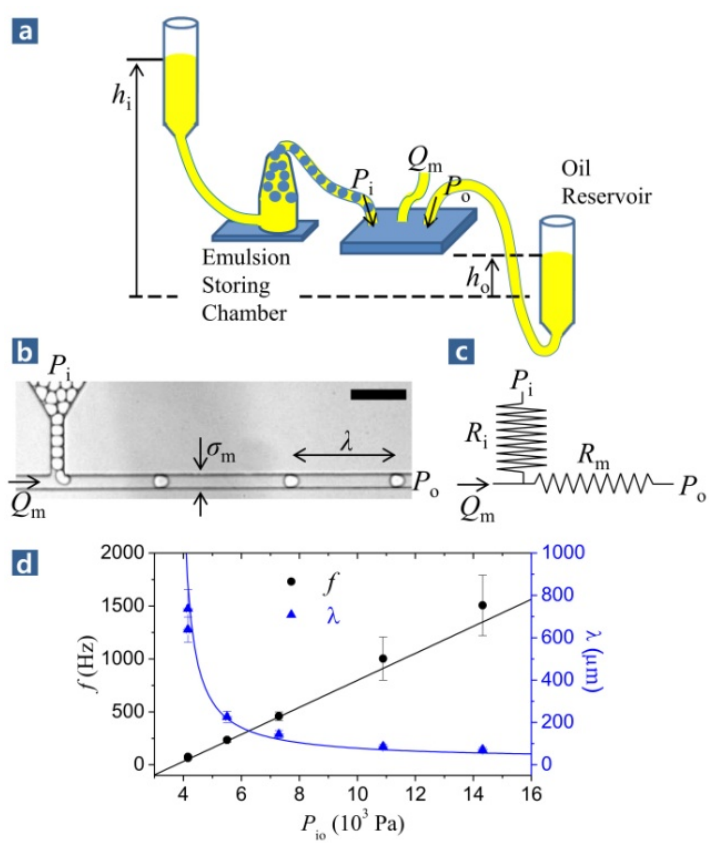

${ }_{35}$ Fig. 2. Reinjection of a single kind of droplet. (a) Apparatus for droplet reinjection. The carrier oil consists of HFE-7500 fluorinated oil with a fluorinated surfactant (surfactant I), and the droplets contain deionized water. (b) Optical micrograph of apparatus. The fluid channels are $25 \mu \mathrm{m}$ high, $30 \mu \mathrm{m}$ wide, and $1.77 \mathrm{~mm}$ long, as measured from the reinjection 40 junction to the collection outlet. The scale bar is $100 \mu \mathrm{m}$ (see ESI $2 \uparrow$ for movie of droplet reinjection). (c) Equivalent electric circuit model corresponding to (b), where the fluidic resistance $R_{\mathrm{m}}$ for the laminar flow is determined from the geometrical dimensions of the channel and the viscosity of the oil (HFE-7500, $1.24 \times 10^{-3} \mathrm{~kg} /(\mathrm{ms})$ ), resulting in ${ }_{45} R_{\mathrm{m}}=1.12 \times 10^{14} \mathrm{~Pa} \mathrm{~s} / \mathrm{m}^{3}$. (d) Injection frequency $(f)$ and droplet-droplet distance $(\lambda)$ as a function of the hydrostatic pressure drop $P_{\text {io }}$. The black curve is a fit to experimental $f$ using Eq. (1a), where $R_{\mathrm{i}}$ and $Q_{\mathrm{m}}$ were used as fitting parameters. Then Eq. (1b) with the same values of $R_{\mathrm{i}}$ and $Q_{\mathrm{m}}$ (blue curve) shows excellent agreement with the experimental $\lambda$.

50

We find that the reinjection frequency $f$ of droplets is linearly proportional to $P_{\text {io }}$, while the droplet-droplet distance $\lambda$ is inversely proportional to the pressure drop $P_{\text {io }}$ for a given flow rate $Q_{\mathrm{m}}$, as shown in Fig. 2(d). These scaling relations agree with 55 those predicted by modelling the device by the equivalent electrical circuit ${ }^{13}$ shown in Fig. 2(c):

$$
\begin{aligned}
& f=f_{\mathrm{o}} \frac{\overline{P_{\mathrm{io}}}-1}{\overline{R_{\mathrm{i}}}+1} \\
& \lambda=\lambda_{0}\left(\frac{\overline{R_{\mathrm{i}}}+1}{\overline{P_{\mathrm{io}}}-1}+1\right)
\end{aligned}
$$

where $f_{0}=Q_{\mathrm{m}} / V_{\mathrm{d}}, \lambda_{0}=V_{\mathrm{d}} / \sigma_{\mathrm{m}}, \overline{R_{\mathrm{i}}}=R_{\mathrm{i}} / R_{\mathrm{m}}$, and $\overline{P_{\mathrm{io}}}=$ ${ }_{60} P_{\mathrm{io}} /\left(R_{\mathrm{m}} Q_{\mathrm{m}}\right)$. Here $V_{\mathrm{d}}$ is the volume of droplet, $\sigma_{\mathrm{m}}$ the crosssectional area of main channel, $R_{\mathrm{i}}$ the fluidic resistance of the droplet channel, and $R_{\mathrm{m}}$ that of the main channel. We can calculate $V_{\mathrm{d}}, \sigma_{\mathrm{m}}$, and $R_{\mathrm{m}}$ from the geometry of the channels, the viscosity of the oil, and the diameter of the droplets, but it is not ${ }_{65}$ trivial to determine $R_{\mathrm{i}}$, owing to the dominant role of interfacial dynamics in the flow of close-packed droplets. Also, there is a significant uncertainty in any estimate of $Q_{\mathrm{m}}$ related to the calibration of the syringe pump. Therefore, we use $R_{\mathrm{i}}$ and $Q_{\mathrm{m}}$ as parameters to fit Eq. (1a) to the experimentally measured $f$, and 70 we find $Q_{\mathrm{m}}=0.12 \mathrm{ml} / \mathrm{h}$ and $\overline{R_{\mathrm{i}}}=3.06 . Q_{\mathrm{m}}$ is close to the value that 
we set on the syringe pump, $0.15 \mathrm{ml} / \mathrm{h}$, and $R_{\mathrm{i}} \approx 3 R_{\mathrm{m}}$ indicates that the resistance for the flow of droplets is much larger than that of the flow of the continuous phase; $R_{\mathrm{i}} \ll R_{\mathrm{m}}$ in the absence of droplets. Using the fitted values of $R_{\mathrm{i}}$ and $Q_{\mathrm{m}}$, we then calculate $\lambda$ 5 from equation (1b) and find excellent agreement with experimental measurements, as shown in Fig. 2(d). This agreement validates our model of the basic physics of droplet reinjection in microfluidics, which follows the usual electric circuit description for laminar flows but with a modified flow 10 resistance for the closed-packed droplets $\left(R_{\mathrm{i}}\right)$.

Two different types of droplets can be reinjected using a device consisting of two opposing T-junctions (a "crossjunction"), as shown in Fig. 3(a), where the oil flow in the middle 15 channel is driven by a syringe pump. The droplets in the upper chamber are driven by hydrostatic pressure $P_{\mathrm{u}}$ and those in the lower chamber by $P_{1}$. To synchronize the reinjection of the droplets, we first tune $P_{\mathrm{u}}$ and $P_{1}$ until the reinjection rates of the two droplet streams are balanced. Then the hydrodynamic 20 coupling between two droplets in opposite channels automatically forces the injected droplets to alternate: when the droplet in the upper chamber starts to emerge into the main flow channel, the pressure on the droplet in the lower chamber increases, holding it in place. The lower droplet therefore emerges only after the upper 25 droplet is released, producing an ordered array of two kinds of droplets, as shown in Fig. 3(a) (see ESI $3 \dagger$ ).

The order of reinjected droplets, however, can change when two droplets come out of the same chamber sequentially (Fig. $3(b)$ ), resulting in what we call a synchronization error. Such 30 errors are quantitatively characterized by counting the number of changes in the order of the droplets and dividing by the total number of droplets injected into the main channel (ESI $1 \dagger$ ). Once the number of order changes as a function of the total number of injected droplets is obtained, the error rate is determined from the 35 slope of the curve.

We find that synchronization errors follow particular patterns. We never observe three sequential droplets emerging from the same chamber, $\{\ldots, \mathrm{U}, \mathrm{U}, \mathrm{U}, \ldots\}$ or $\{\ldots, \mathrm{L}, \mathrm{L}, \mathrm{L}, \ldots\}$, nor do we observe doubly-paired droplets, $\{,, \mathrm{U}, \mathrm{U}, \mathrm{L}, \mathrm{L}, \ldots\}$, where ' $\mathrm{U}$ ' 40 represents a droplet from the upper chamber and ' $L$ ' from the lower chamber. Thus the reinjection ratio of upper to lower droplets is always between $1: 2$ and 2:1, corresponding to droplet configurations $\{\ldots, \mathrm{U}, \mathrm{L}, \mathrm{L}, \mathrm{U}, \mathrm{L}, \mathrm{L}, \ldots\}$ or $\{\ldots, \mathrm{U}, \mathrm{U}, \mathrm{L}, \mathrm{U}, \mathrm{U}, \mathrm{L}, \ldots\}$. In these limiting cases, the error rate is $33 \%$ (black circles in Fig. ${ }_{45} 3(\mathrm{c})$ ). In various experiments with three different sets of devices and droplets, as shown in Fig. 3(a), the error rates are around 10\% and are all less than $33 \%$, as expected (Fig. 3(c)).

The synchronization is the result of a complex interplay between the fluid dynamics of discrete droplets and the 50 continuous flow of oil at the junction. Despite this complexity, we can determine phenomenological rules to minimize the errors. One factor that contributes to synchronization error is a difference in size between droplets from the upper and lower chambers. As observed in Fig. 3(b), an error can occur if the ${ }_{55}$ diameters of the upper and lower droplets are slightly different, reducing the hydrodynamic coupling between them. In the supporting information (ESI $1 \dagger$ ), we show that the error rate increases as the diameter difference increases, even when the pressures $P_{\mathrm{u}}$ and $P_{1}$ are closely matched. Synchronization errors 60 can also occur due to mismatches in these pressures, which are set by the reservoir heights. The balance between $P_{\mathrm{u}}$ and $P_{1}$ (or reservoir heights) can shift over time owing to the relative difference in the reinjection rates and the resulting difference in the volumes of the emulsions remaining in the two reservoirs. ${ }_{65}$ The inset of Fig. 3(c) shows how the error increases 1 hour from the initial reinjection in device III (after 1 hour, one of the chambers was empty). The error can be minimized by regulating $P_{\mathrm{u}}$ and $P_{1}-$ or, equivalently, the heights of the two reservoirs while observing the droplet reinjection in real time.
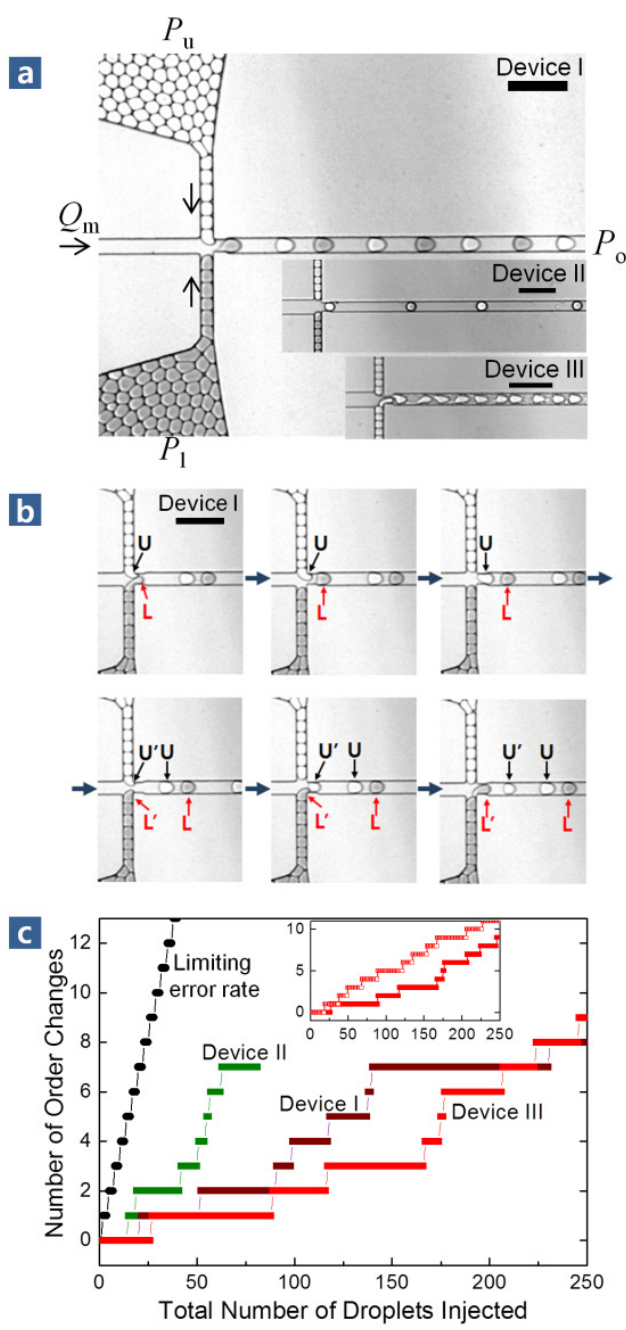

Fig. 3. Synchronized reinjection of two different kinds of droplets. (a) Optical micrographs of the devices. The channels are $25 \mu \mathrm{m}$ high. The width of the main channels downstream of the cross-junction in devices I, 75 II, and III is $30 \mu \mathrm{m}$. The widths of the inlet channels for the oil are $30 \mu \mathrm{m}$ in I, $45 \mu \mathrm{m}$ in II, and $40 \mu \mathrm{m}$ in III. Scale bars: $100 \mu \mathrm{m}$. (b) Optical micrographs showing two sequential droplets coming out of the upper chamber (U, $\left.\mathrm{U}^{\prime}\right)$, resulting in an order change. (c) The number of order changes versus total number of droplets for the three sets of devices and 80 droplets, devices I, II, and III in (a). Black circles indicate order changes for the limiting case of a reinjection ratio of 1:2 (or 2:1) upper droplets to lower droplets. The droplet size distribution, reported as 'mean diameter \pm standard deviation', was measured for 100 droplets reinjected from each chamber of device I, II, and III: $27.9 \pm 1.0 \mu \mathrm{m} \& 28.3 \pm 1.3 \mu \mathrm{m}$ for upper 85 and lower chambers of device I, $26.8 \pm 0.8 \mu \mathrm{m} \& 25.9 \pm 0.7 \mu \mathrm{m}$ for device II, and $23.9 \pm 0.6 \mu \mathrm{m} \& 23.4 \pm 0.6 \mu \mathrm{m}$ for device III. The inset of (c) shows 
the number of order changes for device III after an initial pressure balancing (filled red circles) and 1 hour later (empty red circles). In these experiments $Q_{\mathrm{m}}$ was fixed at values between 0.02 and $0.15 \mathrm{ml} / \mathrm{h}$, and $f$ was between $100 \mathrm{~Hz}$ and $300 \mathrm{~Hz}$.
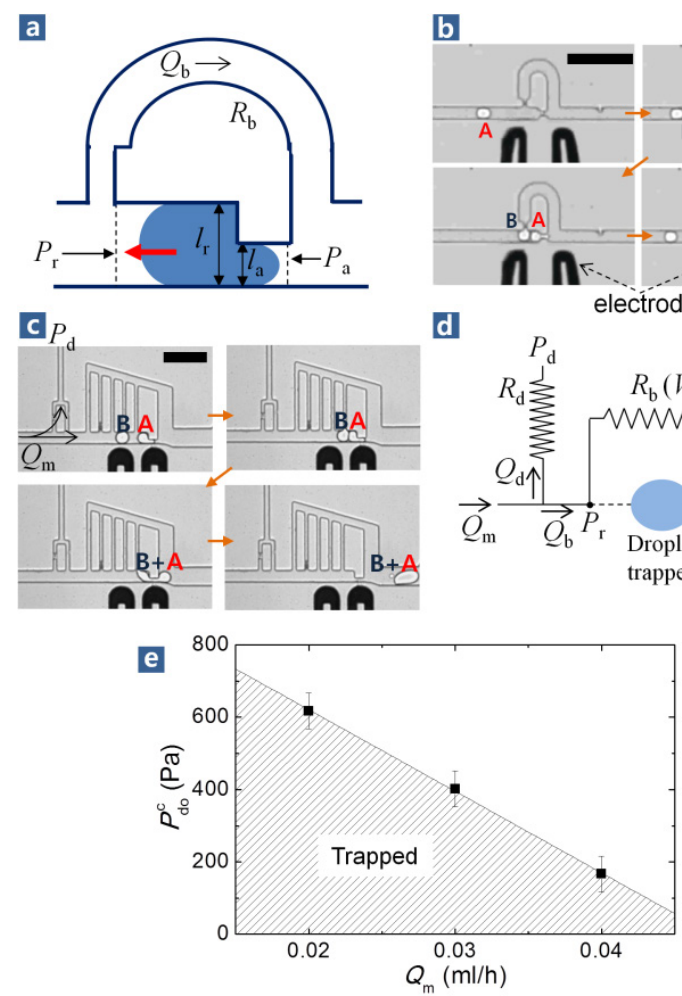

Fig. 4. Coalescence schemes. (a) Schematic of droplet trapped by hydrodynamic and Laplace pressures. (b) Once droplet A is trapped, coalescence occurs when a subsequent droplet (B) meets the trapped 10 droplet and the electrodes (pictured) are held at a constant potential (0.8$1.2 \mathrm{kV})$. (c) General coalescer including a drain channel and multiple bypass channels. (d) Equivalent circuit model for the coalescer in (c). (e) Critical drain pressure $P_{\mathrm{do}}{ }^{\mathrm{c}}$ versus oil flow rate $Q_{\mathrm{m}}$ for the coalescer, showing the range of conditions under which the droplet is trapped. Error 15 bars indicate the uncertainty in $P_{\mathrm{do}}{ }^{\mathrm{c}}$, based on the uncertainty of $\pm 0.5 \mathrm{~cm}$ for the reservoir height. $P_{\mathrm{do}}{ }^{c}$ scales linearly with $Q_{\mathrm{m}}$, as expected from Eq. (2). The fluid channels are $25 \mu \mathrm{m}$ tall, and the scale bars are $100 \mu \mathrm{m}$ in $(\mathrm{b}, \mathrm{c})$.

20 The injected droplets are then fused in a coalescer (Fig. 4), whose design is inspired by droplet-storage devices that use Laplace pressure to trap droplets. ${ }^{14}$ To illustrate the operating principle, we first consider a single droplet sitting at the point where the channel size changes from $l_{\mathrm{r}}$ to $l_{\mathrm{a}}$. The oil is driven at 25 constant flow rate $Q_{\mathrm{b}}$ by a syringe pump (Fig. 4(a)). This sets the pressure drop $P_{\text {ra }}\left(\equiv P_{\mathrm{r}}-P_{\mathrm{a}}\right)=Q_{\mathrm{b}} R_{\mathrm{b}}$ across the droplet, where $R_{\mathrm{b}}$ is the resistance of the bypass channel. If $P_{\text {ra }}$ is smaller than a critical pressure $P_{\mathrm{ra}}{ }^{\mathrm{c}} \sim 2 \gamma / l_{\mathrm{a}}$, where $\gamma$ is the interfacial tension of the droplet in the oil, the droplet is trapped due to the balance of 30 the hydrodynamic and Laplace pressures (red arrow in Fig. 4(a)). When the next droplet (B) enters the coalescer, the two droplets coalesce in a constant electric field generated by applying a voltage of $1 \mathrm{kV}$ across the electrodes, which are spaced about 40 $\mu \mathrm{m}$ apart. ${ }^{4,15}$ Because the combined droplet $(\mathrm{B}+\mathrm{A})$ blocks both 35 the bypass and main channels, it is forced out of the trapping junction to the right, along the route with lower hydrodynamic resistance, as shown in Fig. 4(b). This basic coalescer works when the flow rate is smaller than the critical flow rate given by $P_{\mathrm{ra}} \mathrm{c} / R_{\mathrm{b}}$. Above this rate, droplets flow through the trapping 40 junction without stopping.

While the simple coalescer in Fig. 4(b) accommodates only droplets that fit its trap region, the generalized coalescer shown in Fig. 4(c), which contains multiple bypass channels, accommodates droplets with a range of sizes. Also, the added ${ }_{45}$ drain channel increases the range of flow rates, which is important for high-throughput operation. As modelled in Fig. 4(d), the resistance of multiple bypassing channels $R_{\mathrm{b}}(V)$ is an increasing function of the volume of droplets $V$ in the coalescer. Thus the trap condition $Q_{\mathrm{b}}<P_{\mathrm{ra}}{ }^{\mathrm{c}} / R_{\mathrm{b}}(V)$ also varies with $V$, and for 50 a given $Q_{\mathrm{b}}$, a specific volume (or number) of droplets can be trapped in the coalescer. In other words, for a given $Q_{\mathrm{m}}$ and volume of droplets to be trapped, one can set the drain pressure drop $P_{\mathrm{do}}\left(=P_{\mathrm{d}}-P_{\mathrm{o}}\right)$ so that $Q_{\mathrm{b}}\left(=Q_{\mathrm{m}}-Q_{\mathrm{d}}\right)$ satisfies the condition $Q_{\mathrm{b}}<P_{\mathrm{ra}}{ }^{\mathrm{c}} / R_{\mathrm{b}}(V)$ (see ESI $1 \uparrow$ and $4 \uparrow$ for results on trapping and 55 coalescing more than two droplets by varying $P_{\text {do }}$, demonstrated using a device of the same type). From the circuit model in Fig. 4(d), we calculate the critical value $P_{\mathrm{do}}{ }^{\mathrm{c}}$ below which droplets are trapped for a given $Q_{\mathrm{m}}$ :

$P_{\mathrm{do}}^{\mathrm{c}}=\left(R_{\mathrm{d}} / R_{\mathrm{b}}(V)+1\right) P_{\mathrm{ra}}^{\mathrm{c}}-R_{\mathrm{d}} Q_{\mathrm{m}}$

60 where $R_{\mathrm{d}}$ is the resistance of the drain channel. Equation (2) shows that droplets can be trapped and coalesced at any $Q_{\mathrm{m}}-$ and therefore over a wide range of $f$ and $\lambda($ Eq. $(1 \mathrm{a}, \mathrm{b}))$, or $P_{\mathrm{u}}$ and $P_{1}-$ by controlling $P_{\mathrm{do}}<P_{\mathrm{do}}{ }^{\mathrm{c}}\left(Q_{\mathrm{m}}\right)$ (Fig. 4(e)). Thus a wide range of throughputs, from low to high, are possible on a single chip. We 65 note that the intercept of the curve in Fig. 4(e) provides an estimate of the interfacial tension between the droplet and oil, $\gamma \sim$ $\left(l_{\mathrm{a}} / 2\right) P_{\mathrm{ra}}{ }^{\mathrm{c}} \sim\left(l_{\mathrm{a}} / 2\right) P_{\mathrm{do}}{ }^{\mathrm{c}}\left(Q_{\mathrm{m}}=0\right)=3.7 \times 10^{-3} \mathrm{~N} / \mathrm{m}$ with $l_{\mathrm{a}}=10 \mu \mathrm{m}($ Fig. $4(\mathrm{c}))$, which is consistent with the independently measured value $\gamma_{\exp }=4.1 \times 10^{-3} \mathrm{~N} / \mathrm{m}$ (see ESI $\left.1 \dagger\right)$.

70

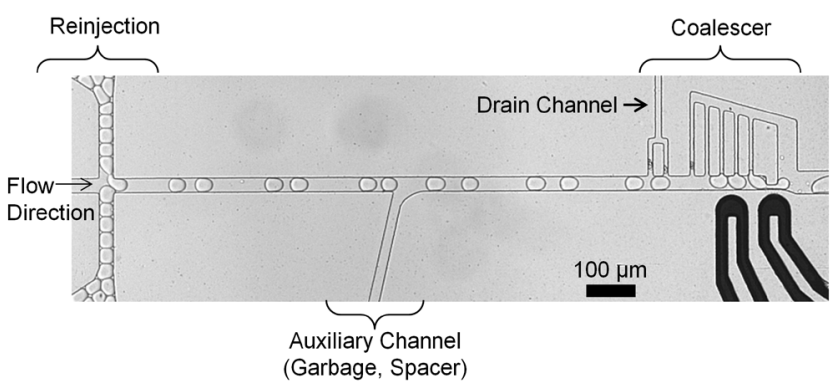

Fig. 5. Apparatus showing both synchronized reinjection and coalescence of droplets (see ESI $5 \dot{\dagger}$ ). Clogs can be removed by reducing the outlet pressure of the auxiliary channel. A droplet injection frequency of $75 \sim 200 \mathrm{~Hz}$ was used for this experiment. The fluid channels are $25 \mu \mathrm{m}$ tall and the scale bar is $100 \mu \mathrm{m}$.

Finally we demonstrate a device that incorporates both reinjection and coalescence (Fig. 5). We add an auxiliary channel 80 to remove any debris that might clog the channel at the initial stage of reinjection. The pressure in the auxiliary channel can also be varied to control the droplet-droplet distance. From the video analysis, we find that the reinjection error $\mathrm{E}=13 \%$ and the 
coalescence yield $\mathrm{Y}=85 \%$ for this particular device. The difference of $2 \%$ between $\mathrm{E}$ and $1-\mathrm{Y}$ comes from the error in the coalescer, which allows some single droplets to pass through (see ESI $5 \dagger$ ). Coalescer errors can occur if the oil flow in the main 5 channel increases momentarily, violating the trap condition, $P_{\mathrm{ra}}=Q_{\mathrm{b}} R_{\mathrm{b}}<P_{\mathrm{ra}}{ }^{\mathrm{c}} \sim 2 \gamma / l_{\mathrm{a}}$ (Fig. 4(a)). Specifically, when a droplet is released into the main channel, it plugs the channel momentarily, which causes a pressure fluctuation. ${ }^{16}$ The resulting increase in $Q_{\mathrm{b}}$ can push a droplet out of the coalescence junction.

10 About half of the error in reinjection comes from the slight variation in droplet diameter, as seen in ESI $5 \uparrow$. Uniformly-sized droplets should therefore sharply reduce the overall error rate. These can be produced by using appropriate surfactants and flow rates to prevent satellite droplets from forming during droplet 15 creation and keep droplets from breaking or coalescing during reinjection.

\section{Conclusions}

The method we have presented for reinjecting and coalescing droplets should be compatible with a wide variety of on-chip 20 applications. For instance, two emulsions with droplets containing single cells could pass through a sorter and then be reinjected and coalesced in our device for the study of cell-cell interactions. ${ }^{17}$ We plan to use the method to create droplets with controlled numbers of various kinds of Brownian particles to 25 study colloidal self-assembly.

\section{Acknowledgements}

We are grateful to Tina Lin, Dr. Shin-Hyun Kim and Dr. Assaf Rotem for invaluable discussions. This work was supported by the Harvard MRSEC under National Science Foundation award 30 number DMR-0820484, an Alfred P. Sloan fellowship, the German Research foundation (Sp 1282/1-1), and the National Research Foundation of Korea Grant funded by the Korean Government [NRF-2010-357-C00036].

\section{${ }_{35}$ Notes and references}

${ }^{a}$ Department of Physics, Harvard University, Cambridge, Massachusetts 02138, USA. Fax: +1 617495 0416; Tel: +1 617495 3763; E-mail: vnm@seas.harvard.edu

${ }^{b}$ School of Engineering and Applied Sciences, Harvard University, 40 Cambridge, Massachusetts 02138, USA.

${ }^{c}$ College of Engineering, Temple University, Philadelphia, 19122, USA.

${ }^{d}$ Korea Research Institute of Chemical Technology, Daejeon 305-600,

Korea.

${ }^{e}$ Department of Polymer Science and Engineering, Sungkyunkwan

45 University, University, Suwon, 440-746, Korea.

${ }^{\S}$ Current address: S. LSI, Giheung-campus, Samsung electronics, Yonginsi, 446-811, Korea.

\# Current address: Institut für Mikrotechnik Mainz GmbH, Carl-ZeissStraße 18-20, 55129 Mainz, Germany.

50

$\dagger$ Electronic Supplementary Information (ESI) available: Supporting information (ESI 1) and movies (ESI 2-5). See DOI: 10.1039/ C3LC51214B/

55

1 M. Margulies et al., Nature, 2005, 437, 376-380.

2 A. R. Abate, T. Hung, P. Mary, J. J. Agresti, and D. A. Weitz, Proc. Natl. Acad. Sci. USA, 2010, 107, 19163-19166.
3 G.-R. Yi et al., Adv. Mater., 2003, 15, 1300-1304.

604 K. Ahn et. al., Appl. Phys. Lett., 2006, 88, 264105.

5 C. N. Baroud, M. R. S. Vincent and J.-P. Delville, Lab on a Chip, 2007, 7, 1029-1033.

6 D. R. Link et al., Angew. Chem. Int. Ed., 2006, 45, 2556 -2560.

7 N. Bremond, A. R. Thiam, and J. Bibette, Phys. Rev. Lett., 2008, 100, 024501.

8 X. Niu, S. Gulati, J. B. Edel and A. J. deMello, Lab on a Chip, 2008, 8, 1837-1841.

9 M. Prakash and N. Gershenfeld, Science, 2007, 315, 832-835.

10 M. Schindler and A. Ajdari, Phys. Rev. Lett., 2008, 100, 044501.

7011 Y. Xia and G. M. Whitesides, Annu. Rev. Mater. Sci. 28, 153 (1998).

12 Y. J. Kang and S. Yang, Lab on a Chip, 2012, 12, 1881-1889.

13 K. W. Oh, K. Lee, B. Ahna and E. P. Furlanib, Lab on a Chip, 2012, 12, 515-545.

14 H. Boukellal, S. Selimovic, Y. Jia, G. Cristobalb and S. Fraden, Lab on a Chip, 2009, 9, 331-338.

15 M. Chabert, K. D. Dorfman and J. L. Viovy, Electrophoresis, 2005, 26, 3706-3715.

16 P. A. Romero and A. R. Abate, Lab on a Chip, 2012, 12, 5130-5132.

17 D. C. Kirouac et al., Mol. Syst. Biol., 2009, 5, 293-312. 


\title{
"Synchronized reinjection and coalescence of droplets in microfluidics"
}

\author{
Supplementary Information
}

\author{
Manhee Lee,${ }^{a, \S}$ Jesse W. Collins,${ }^{b}$ Donald M. Aubrecht, ${ }^{b}$ Ralph A. Sperling, ${ }^{b, \#}$ Laura Solomon, ${ }^{c}$ \\ Jong-wook Ha, ${ }^{d}{ }^{\text {Gi-Ra Yi, }}{ }^{e}$ David A. Weitz ${ }^{a, b}$, and Vinothan N. Manoharan* ${ }^{*, a}$ \\ ${ }^{a}$ Department of Physics, Harvard University, Cambridge, Massachusetts 02138, USA. Fax: +1 617 495 0416; Tel: +1 617 495 3763; E- \\ mail:vnm@seas.harvard.edu \\ ${ }^{b}$ School of Engineering and Applied Sciences, Harvard University, Cambridge, Massachusetts 02138, USA. \\ ${ }^{c}$ College of Engineering, Temple University, Philadelphia, 19122, USA. \\ ${ }^{d}$ Korea Research Institute of Chemical Technology, Daejeon 305-600, Korea. \\ ${ }^{e}$ Department of Polymer Science and Engineering, Sungkyunkwan University, University, Suwon, 440-746 Korea. \\ ${ }^{\S}$ Current address: S. LSI, Giheung-campus, Samsung electronics, Yonginsi, 446-811, Korea. \\ \# Current address: Institut für Mikrotechnik Mainz GmbH, Carl-Zeiss-Straße 18-20, 55129 Mainz, Germany.
}

\section{Synthesis of Fluorinated Surfactants}

In the experiments, we synthesized and used two fluorinated surfactants [S1], surfactant I and surfactant II. Surfactant I was used in most experiments except the experiments using device III shown in Fig. 3(a,c). Here we describe the experimental protocol to synthesize surfactant I and II.

(1) Synthesis of Surfactant I

In a typical reaction, we dissolved $80 \mathrm{~g}$ of commercially available perflouro-polyether Krytox 157-FSH (DuPont, $12.3 \mathrm{mmol}$ assuming $6500 \mathrm{~g} / \mathrm{mol}$ ) in an equal volume of HFE-7100 (3M) in a round flask and activated its terminal carboxylic group with a 10x molar excess of oxalyl chloride (10.4 mL, Sigma-Aldrich). The mixture became hazy and slightly yellow and was stirred overnight. Then, the solvent and unreacted oxalyl chloride were distilled off $\left(70{ }^{\circ} \mathrm{C}\right.$ and increased vacuum) and neutralized by bubbling the vapors through $2 \mathrm{M}$ $\mathrm{KOH}$. Then, the flask was attached to a rotation evaporator to remove all remaining traces of unreacted oxalyl chloride. The activated Krytox was allowed to cool down before diluting it in $50 \mathrm{~mL}$ HFE-7100, as all solvent had been evaporated in the previous process.

For the central hydrophilic block of the surfactant, a diamine PEG was used (Jeffamine ED900: O,O'bis(aminopropyl) polypropylene glycol-b-polyethylene glycol-b-polypropylene glycol, $\mathrm{x}+\mathrm{z} \sim 6.0, \mathrm{y} \sim 12.5$, a gift from Huntsman Chemicals), which yields one amino group with a few PPO units at each end. A stock solution was prepared by dissolving $6.6 \mathrm{~g}(14.8 \mathrm{mmol})$ in $100 \mathrm{ml}$ anhydrous dichloromethane, and $50 \mathrm{ml}$ HFE-7100 were added. The total amount of Jeffamine yields a 1.2x molar excess in regard the activated carboxylic groups from Krytox-158 FSH, assuming $6500 \mathrm{~g} / \mathrm{mol}$. Due to the presumably polydisperse reagents and the uncertainity of the number of molecules present, the stock solution was added stepwise to first favor the formation of triblock molecules with an excess of Krytox (assuming the nominal molecular weights), and then to saturate all remaining Krytox molecules. Both di- and triblock molecules, and even the plain Krytox with its carboxylic group, are surface-active molecules and effective in stabilizing the droplets.

Of the Jeffamine ED900 stock solution, $100 \mathrm{ml}$ were added to the flask containing the activated Krytox to initiate the reaction, after $30 \mathrm{~min}$ another $25 \mathrm{ml}$ were added, after another $30 \mathrm{~min}$ the remaining $25 \mathrm{ml}$. During the reaction, the flask was attached to the rotation evaporator, briefly heated after each addition, and then kept stirring at room temperature to avoid complete evaporation of the solvents. After rotating overnight, the temperature was set to $70{ }^{\circ} \mathrm{C}$ and vacuum was applied to evaporate all solvent. The final product was a milkywhite viscous oil.

The material was diluted in $\sim 50 \mathrm{ml}$ HFE-7100 and collected into several $50 \mathrm{ml}$ plastic centrifuge tubes (VWR, high g). A table spoon of plain PEG powder (100,000 g/mol, Sigma-Aldrich) was added to each tube and mixed with the samples by shaking. After centrifugation overnight, the sample separated into a clear bottom phase and a white top layer consisting of the excess of unreacted Jeffamine. The PEG powder helps to form a solid plug of upper phase that does not get redispersed too easily in the following processing step. With a sharp razor, we carefully cut into the bottom of the plastic tube and collected the clear fluorinated bottom fraction into a new tube. Residual material and turbid fractions were pooled and centrifuged again. After evaporation of the 
solvent at $65^{\circ} \mathrm{C}$ over two days, the obtained material was viscous and clear, with a yellowish shade (Fig. S1.).

\section{(2) Synthesis of Surfactant II}

Perfluoropolyether (PFPE) block was first synthesized from the alkali metal fluoride catalyzed anionic oligomerization of hexafluoropropylene oxide (HFPO, 3M/Dyneon). In a $300 \mathrm{~mL}$ jacketed autoclave equipped with pressure gauge, thermocouple, inlet and outlet valves, $0.73 \mathrm{~g}$ of spray-dried solid potassium fluoride (KF, Sigma-Aldrich) and $15.0 \mathrm{~g}$ of tetraethylene glycol dimethyl ether (Sigma-Aldrich) were charged initially, and then the reactor was evacuated and filled with high purity nitrogen. Typically, three vacuum/nitrogen cycles were performed, and the pressure inside the reactor was adjusted to equilibrate with atmospheric condition with nitrogen. The suspension of KF was stirred for 1 hour at $25{ }^{\circ} \mathrm{C}$. A cross-type magnetic spin bar was used to agitate the reaction media. $303.1 \mathrm{~g}$ of gaseous HFPO were introduced into the reactor during 6 hours. After finishing the addition of HFPO, the reaction mixture was stirred for sufficient time (typically for 2 hours) in order to consume almost all of the HFPO. The oligomerization product was transferred into a separation funnel, and the organic solvent layer was discarded after phase separation. The HFPO oligomer with acid fluoride end group was filtered and analyzed by gas chromatography (Younglin, M600D) in order to determine the molecular weight.

The stabilizer consisting of fluorophilic-fluorophobic-fluorophilic blocks was synthesized as follows. $30 \mathrm{~g}$ (50 mmol) of O,O'-bis(aminopropyl) polypropylene glycol-b-polyethylene glycol-b-polypropylene glycol (Mn 600, Jeffamine ED600, $\mathrm{x}+\mathrm{z} \sim 3.6, \mathrm{y} \sim 9.0$ ) was diluted in $90 \mathrm{~g}$ of Asahiklin AK-225 (Asahi Glass Company) solvent at room temperature. To this, a slight excess $(100 \mathrm{~g}, 106 \mathrm{mmol})$ of PFPE (Mn 930, n 3.7) was slowly added with vigorous stirring. The formation of an amide linkage between amine and acid fluoride could be confirmed by FT-IR (Jasco FT/IR4000). After completion of the reaction, the crude product was washed in a $\mathrm{NaHCO}_{3}$ aqueous solution twice and with deionized water until the $\mathrm{pH}$ of the aqueous phase was neutral. Finally, the PFPE-based stabilizer was recovered from solution by evaporating the solvent at reduced pressure. $107 \mathrm{~g}$ of yellowish viscous product were obtained (Fig. S1.).
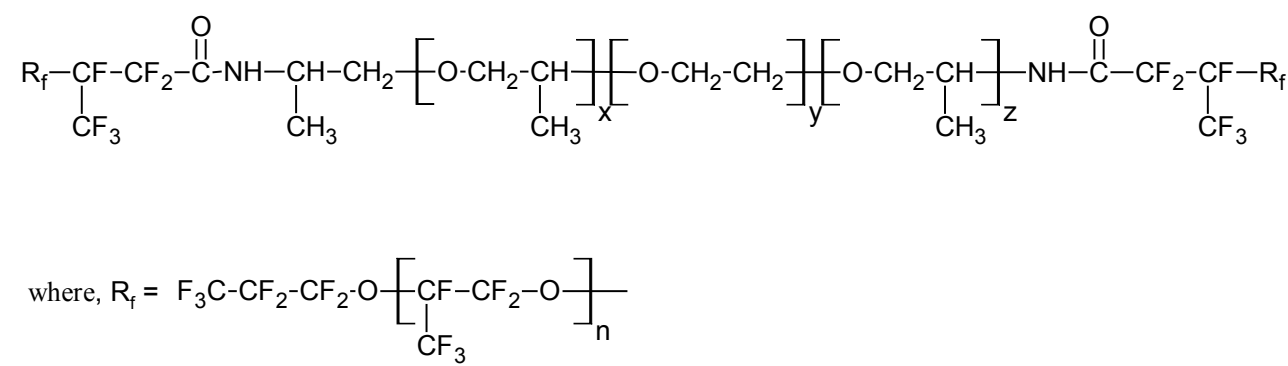

Fig. S1. Chemical structure of the PFPE-PEG-PFPE surfactants: Surfactant I: $\mathrm{n} \sim 37, \mathrm{x}+\mathrm{z} \sim 6.0, \mathrm{y} \sim 12.5$. Surfactant II: $\mathrm{n}=$ $3.7, \mathrm{x}+\mathrm{z} \sim 3.6, \mathrm{y} \sim 9.0$.

The molecular weight of the synthesized oligomers was also measured by chromatography (Fig. S2(a)). Gas chromatography (GC) analysis was performed with M600D GC system (Younglin Instrument, Korea) equipped with OV-1 capillary column $(60 \mathrm{~m} \times 0.25 \mathrm{~mm}$ ID $\times 0.25 \mu \mathrm{m}$, Ohio Valley Specialty Company, USA $)$ and FID detector. The detector and injector temperatures were set at $280{ }^{\circ} \mathrm{C}$. The oven temperature was programmed for $40{ }^{\circ} \mathrm{C}$ for $5 \mathrm{~min}$, and reached $240{ }^{\circ} \mathrm{C}$ with a heating rate of $20{ }^{\circ} \mathrm{C} \mathrm{min}{ }^{-1}$ where it stayed for $20 \mathrm{~min}$. In order to enhance the peak resolution, the acid fluoride end groups were transformed into methyl ester by reacting with methanol. After mixing the HFPO oligomer with a small portion of methanol in a vial, the by-product of HF and excess methanol were removed by washing with deionized water. The resulting PFPE methyl ester was treated by anhydrous magnesium sulfate and then filtered through a syringe filter before analysis. Finally, the PFPE methyl ester was diluted with 1,1,2-trichlorotrifluoroethane (Freon-113). Because the GC analysis provides the weight-averaged molecular weight, it is possible to estimate the polydispersity index $\left(M_{\mathrm{w}} / M_{\mathrm{n}}\right)$ of PFPEs from the following relationships: $M_{\mathrm{n}}=\sum_{i} n_{i} M_{i} / \sum_{i} n_{i}$ and $M_{\mathrm{w}}=\sum_{i} n_{i} M_{i}^{2} / \sum_{i} n_{i} M_{i}$, where $n_{\mathrm{i}}$ represents the GC area percent and $M_{\mathrm{i}}$ is molar mass of the $i$-mer. The synthesized PFPE showed $M_{\mathrm{n}} \sim 940$ and $M_{\mathrm{w}} \sim 960$.

The FT-IR spectrum of PFPE (Fig. S2(b)) showed two characteristic peaks denoting two different end groups: acid fluoride (COF, $\left.1885 \mathrm{~cm}^{-1}\right)$ and carboxylic acid $\left(\mathrm{COOH}, 1778 \mathrm{~cm}^{-1}\right)$, but this might have resulted from rapid reaction of acid fluoride with atmospheric moisture during the sample preparation and analysis. Neat Jeffamine ED 600 shows $\mathrm{NH}$ bending and stretching vibrations at $1594 \mathrm{~cm}^{-1}$ and $3350 \mathrm{~cm}^{-1}$. In the IR spectrum of the 
synthesized stabilizer, new peaks appear at $1716 \mathrm{~cm}^{-1}$ and $1548 \mathrm{~cm}^{-1}$, representing $\mathrm{C}=\mathrm{O}$ stretching of the carbonyl group and amide II band. $\mathrm{CH}_{2}$ stretching and scissoring bands at 2860 and $1450 \mathrm{~cm}^{-1}$ originate from Jeffamine. There is no indication of unreacted PFPE.

(a)

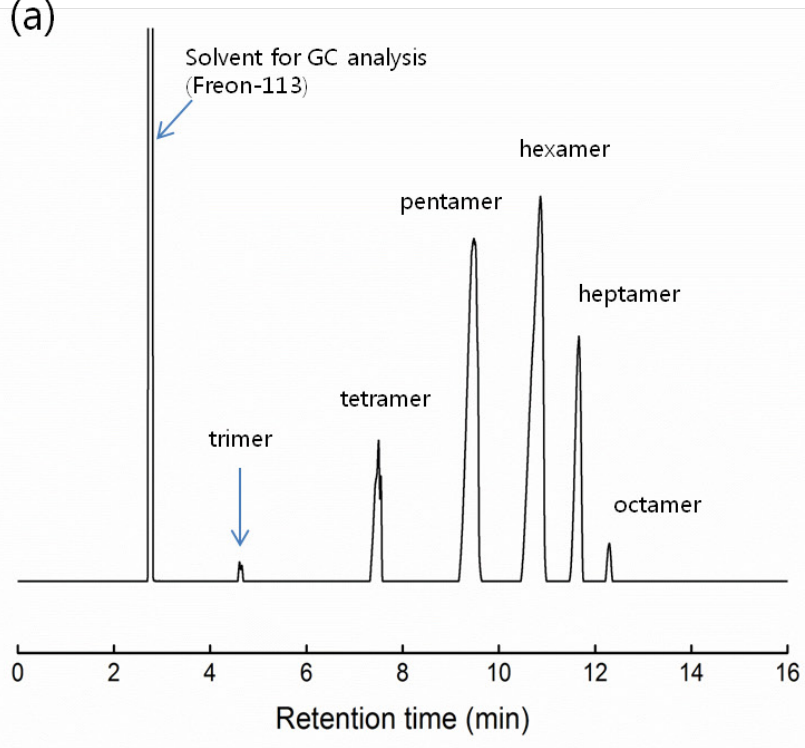

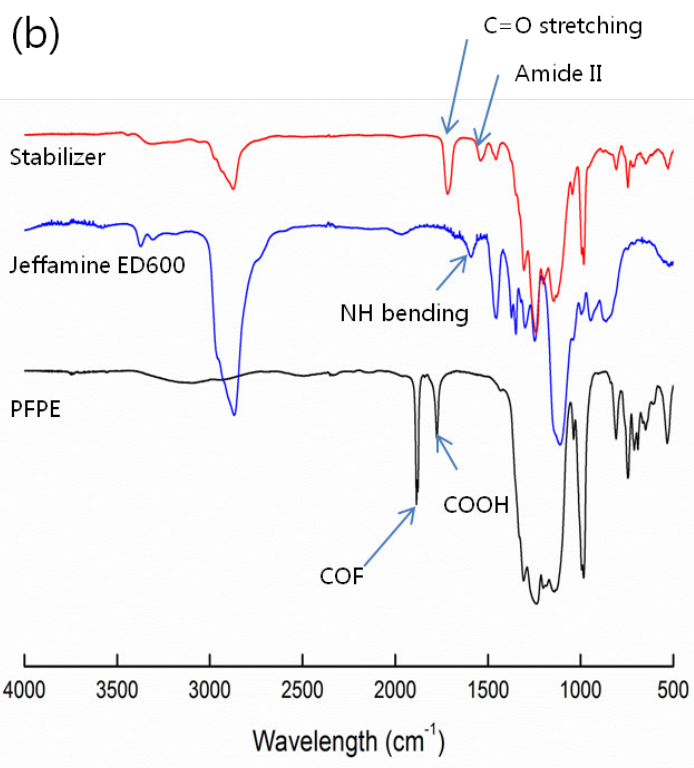

Fig. S2. (a) Gas Chromatography of PFPE (HFPO oligomer). (b) FT-IR analysis of PFPE surfactant.

\section{Measurement of Oil-Water Interfacial Tension}

Interfacial tensions play a key role in the formation, reinjection, and coalescence of droplets in our experiments. In particular, the coalescer in our study requires holding one droplet at the coalescence junction until the other droplet comes and touches it. The droplet is held at the junction under the condition, $Q_{\mathrm{b}} R_{\mathrm{b}}<P_{\mathrm{ra}}{ }^{\mathrm{c}} \sim 2 \gamma / l_{\mathrm{a}}$, as described in the manuscript. Here we present the simple microfluidic method we used to measure the interfacial tension between oil and water by using hydrostatic pressure in conjunction with a conventional syringe pump.

Figure S3(a) shows the microfluidic tensiometer, operated by two hydrostatic pressures $P_{\mathrm{i}}$ and $P_{\mathrm{o}}$ and one syringe pump. We inject the oil (HFE-7500) containing the desired surfactant into the device at a constant flow rate $Q_{\mathrm{m}}$ using a syringe pump while applying hydrostatic pressures $P_{\mathrm{i}}$ to upper chamber filled with the aqueous phase (DI-water) and $P_{\mathrm{o}}$ to the outlet of the device. By modelling the fluidic system with an equivalent circuit, we obtain a simple relation between $P_{\text {io }}\left(=P_{\mathrm{i}}-P_{\mathrm{o}}\right)$ and the curvature of the aqueous phase $r_{\mathrm{L}}$ (red arrow in Fig. S3(a).),

$$
P_{\text {io }}=\gamma\left(\frac{1}{r_{\mathrm{L}}}+\frac{1}{h}\right)+R_{\mathrm{m}} \mathrm{Q}_{\mathrm{m}}
$$

where $\gamma$ is the interfacial tension, $h$ the height of the channel, and $R_{\mathrm{m}}$ the fluidic resistance of the main channel indicated in Fig. S3(a). For a given $Q_{\mathrm{m}}, r_{\mathrm{L}}$ varies depending on $P_{\mathrm{io}}$. Once we get a curve of $P_{\text {io }}$ as a function of $1 / r_{\mathrm{L}}, \gamma$ is then simply given by the slope of the curve. Figure S3(b) shows the experimentally measured $P_{\text {io }}$ and $1 / r_{\mathrm{L}}$ when using the oil (HFE-7500) and surfactant I. We get $\gamma=3.7 \times 10^{-3} \mathrm{~N} / \mathrm{m}$ from the slope of the linear fit (black curve in Fig. 2(b)). We also used the traditional pendant-drop method to measure $\gamma$. Since the density of water $(1.0 \mathrm{~g} / \mathrm{ml})$ is very different from that of the oil $(1.6 \mathrm{~g} / \mathrm{ml})$, water drops were often ruptured during the operation, preventing an accurate measurement. Still, we find an interfacial tension on the order of $5 \times 10^{-3} \mathrm{~N} / \mathrm{m}$. 

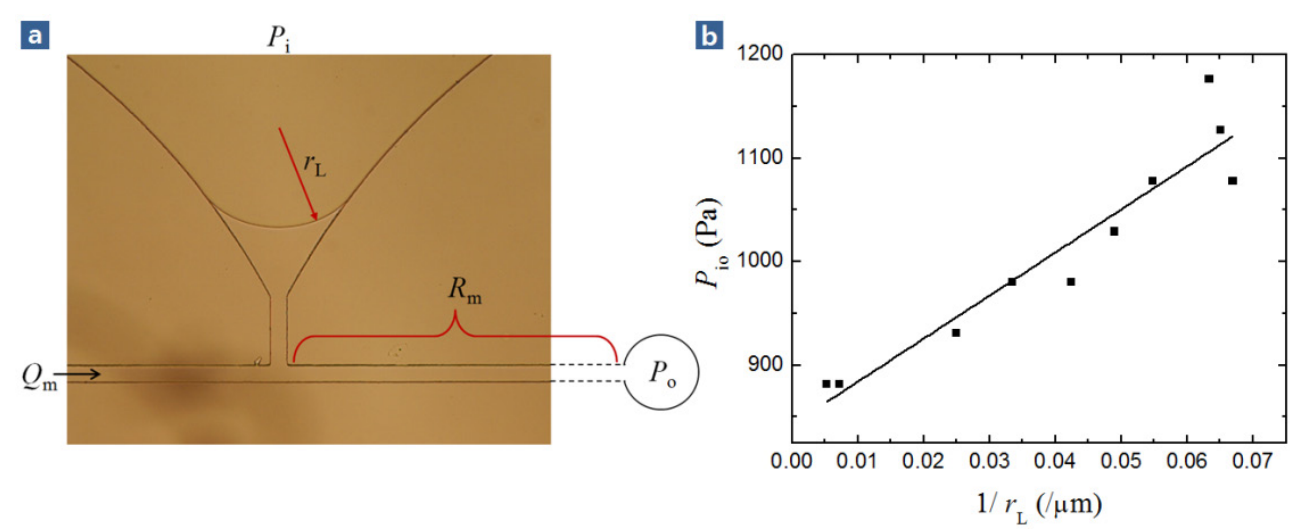

Fig. S3. Measurement of interfacial tension using microfluidic tensiometer. (a) Optical micrograph of our microfluidic tensiometer, which consists of a constant flow of surfactant-dissolved oil driven by a syringe pump, with the aqueous phase in the upper chamber subject to hydrostatic pressure $P_{\mathrm{i}}$, and the outlet to pressure $P_{\mathrm{o}}$. (b) Experimentally measured $P_{\text {io }}\left(=P_{\mathrm{i}}\right.$ $P_{\mathrm{o}}$ ) as a function of $1 / r_{\mathrm{L}}$ and linear fit using Eq. (S1)

\section{Yield Analysis}

The final yield of reinjection and coalescence of droplets depends on the initial orderings of droplets reinjected. Let us represent the array of droplets as an ordered set $\mathrm{S}=\{a(1), a(2), \ldots, a(\mathrm{~N})\}$, where $\mathrm{N}$ is the total number of droplets injected and $a(\mathrm{i})$ is defined as 1 if a droplet came out from the upper chamber or 0 if otherwise. Then, the number of wrong coalescences (W), coalescence of droplets from same chamber, is given as $\mathrm{W}=$ $\sum_{\mathrm{i}=1}^{\mathrm{N} / 2} \delta_{a(2 \mathrm{i}-1), a(2 \mathrm{i})}$ where $\delta_{i j}$ is the Kronecker Delta. The number of order changes (NOC) of reinjection defined in the manuscript is represented as $\mathrm{NOC}=\sum_{\mathrm{i}=1}^{\mathrm{N}} \delta_{a(\mathrm{i}), a(\mathrm{i}+1)}$, which can be rearranged as $\mathrm{NOC}=\sum_{\mathrm{i}=1}^{\mathrm{N} / 2} \delta_{a(2 \mathrm{i}-1), a(2 \mathrm{i})}+\sum_{\mathrm{i}=1}^{\mathrm{N} / 2} \delta_{a(2 \mathrm{i}), a(2 \mathrm{i}+1)}$. If we assume translation symmetry in the droplet array, the second part becomes the same as the first part of the rearranged NOC (by $2 \mathrm{i} \rightarrow 2 \mathrm{i}+1$ ), and thus $\mathrm{E}=\mathrm{NOC} / \mathrm{N}=2 \mathrm{~W} / \mathrm{N}$. From the definition of yield $(\mathrm{Y}), \mathrm{Y} \equiv(\mathrm{N} / 2-\mathrm{W}) /(\mathrm{N} / 2)$, we finally get $\mathrm{Y}=1-\mathrm{E}$.

\section{Synchronized Reinjection of Droplets and Their Size Distributions}

There are many factors that affect the synchronized reinjection in our devices, including the size distribution of droplets, the externally applied hydrostatic pressures, and material properties such as the interfacial tension and viscosity in the upper and lower chambers (Fig. 3(a) in the manuscript). For the size-distribution, it is obvious that if the sizes of the two droplets that meet at the junction are very different, then the injection of droplets does not alternate. One may therefore expect that the synchronized reinjection of droplets becomes worse as the mean diameters of the droplets in the two chambers differ more. In this section, we present a preliminary data supporting this conjecture.

Figure S4 shows a set of three experiments using device I with emulsions I, II and III. Each emulsion consists of two kinds of droplets; droplets made with DI-water in the upper chamber and droplets made with DI-water containing dye (Allura Red, SigmaAldrich) in the lower chamber. They were prepared in the same way using flow-focusing and were reinjected into device I. Through image analysis of optical micrographs, we investigated the diameter distributions of close-packed droplets in the upper and lower chambers of the device before they were released into the main flow channels. We find that the error rate increases as the difference in the mean diameters of the droplets in the upper (red bars) and lower (black bars) chambers increases, even when balancing the pressure $P_{\mathrm{u}}$ and $P_{\mathrm{l}}$, (Fig. 3(a) in the manuscript) as well as possible (Fig. S4 and Table S1). 

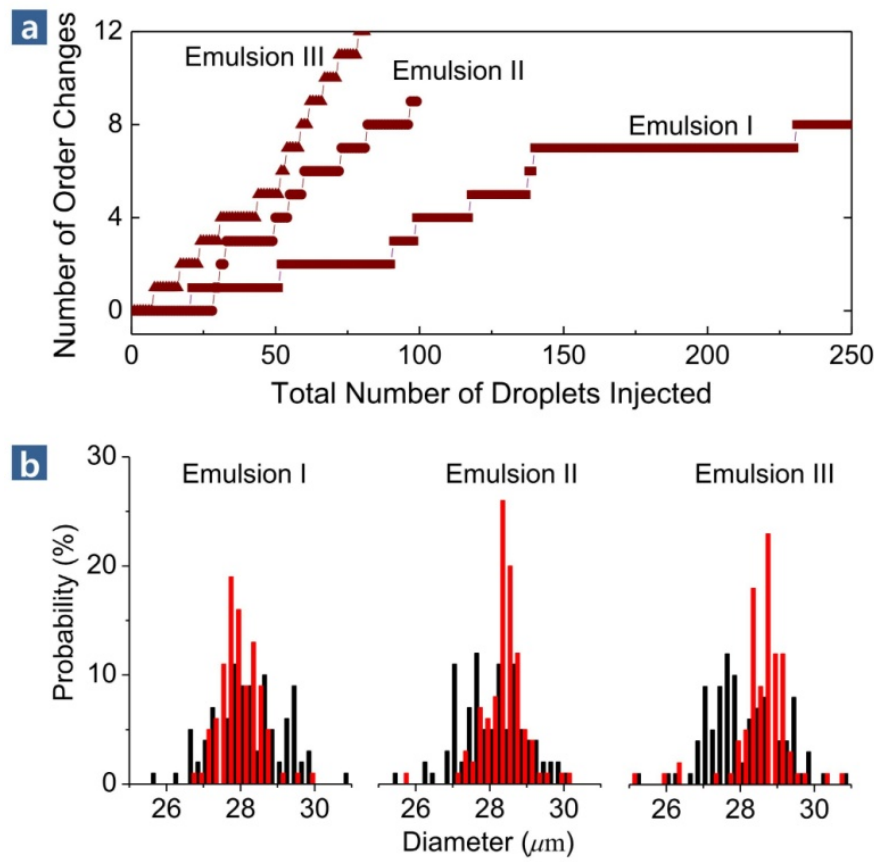

Fig. S4. Error rates in reinjecting droplets and their diameter distributions. (a) Number of order changes as a function of total number of droplets injected for emulsions I, II, and III using device I. (b) Diameter distributions of droplets in upper (red bars) and lower (black bars) chambers. We selected 100 droplets in each chamber and determined the diameter by taking optical micrographs and counting the number of pixels in each droplet.

\begin{tabular}{ccccccc} 
& \multicolumn{2}{c}{ Emulsion I } & \multicolumn{2}{c}{ Emulsion II } & \multicolumn{2}{c}{ Emulsion III } \\
& Upper Chamber & Lower Chamber & $\begin{array}{c}\text { Upper } \\
\text { Chamber }\end{array}$ & $\begin{array}{c}\text { Lower } \\
\text { Chamber }\end{array}$ & $\begin{array}{c}\text { Upper } \\
\text { Chamber }\end{array}$ & $\begin{array}{c}\text { Lower } \\
\text { Chamber }\end{array}$ \\
$\begin{array}{c}\text { Mean Diameter } \\
(\mu \mathrm{m})\end{array}$ & 27.9 & 28.3 & 28.3 & 27.8 & 28.8 & 28.0 \\
$\begin{array}{c}\text { Standard } \\
\text { Deviation }(\mu \mathrm{m})\end{array}$ & 1.0 & 1.3 & 0.9 & 1.5 & 1.7 & 1.2
\end{tabular}

Table S1. Statistics of droplet diameters for emulsions I, II and III in Fig. S4(b).

\section{Trap and Coalescence of Multiple Droplets (See also ESI 4)}

In the manuscript, we demonstrated a coalecer that traps one droplet and fuses the trapped droplet with another droplet. For a given droplet volume $(V)$ and flow rate of the carrier oil $\left(Q_{\mathrm{m}}\right)$ we achieved coalescence by regulating the drain pressure $P_{\mathrm{do}}$. In this section, we show that this scheme works also for trapping and coalescencing multiple droplets, which was also realized experimentally as shown in ESI 4.

Let us first consider Eq. (2) in the manuscript, where $R_{\mathrm{b}}(V)$ is an increasing function of the droplet volume $V$. By replacing $V$ by $2 V$ and $3 V$, we can plot two other curves from Eq. (2) with $R_{\mathrm{b}}(2 V)$, and similarly Eq. (2) with $R_{\mathrm{b}}(3 V)$, which have lower intercept points (see Eq. (2) of the manuscript). Such curves can be found experimentally, but in the cartoon shown in Fig. S4 we present two hypothetical curves for explanatory purposes. The plot shows that one can easily find a specific regime in which a specific number of droplets become trapped.

For example, when operating under a condition $Q_{\mathrm{m}}=Q$ and $P_{\mathrm{do}}=P$ (black circle in Fig. S5), a droplet with volume $V$ that comes into the coalescer is trapped, because the condition satisfies the trap condition, $P_{\mathrm{do}}<$ $P_{\text {do }}^{\mathrm{c}}(V)$ (black curve in Fig. S5; see manuscript for details). When the next droplet comes in, the two droplets are coalesced into a single droplet with volume $2 V$ under an electric-field. From the diagram, we find that the big droplet with volume $2 \mathrm{~V}$ cannot be trapped and therefore exits the coalescer, because $P_{\mathrm{do}}>P_{\text {do }}^{\mathrm{c}}(2 \mathrm{~V})$. Now let us 
decrease the drain pressure to $P_{\mathrm{do}}=P^{\prime}$ at the fixed $Q_{\mathrm{m}}=Q$. The new operating condition, marked as a blue circle in Fig. S5, lies in $P_{\text {do }}^{\mathrm{c}}(3 V)<P_{\text {do }}<P_{\text {do }}^{\mathrm{c}}(2 V)$, and therefore the regime results in two droplets being trapped and three droplets coalescenced. The trapping and coalescence of a controlled number of droplets by varying $P_{\mathrm{do}}$ is demonstrated in ESI 4 experimentally and can in principle be extended to coalesce more than three droplets.

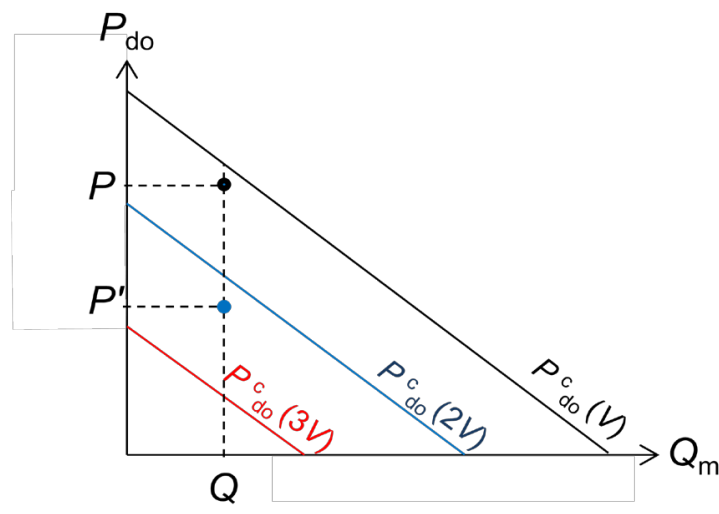

Fig. S5. State diagram of trap and coalescence of multiple droplets for the coalescer shown in Fig. 4(c) of the manuscript. The black curve represents Eq. (2) with $R_{\mathrm{b}}(V)$ (as shown in the manuscript), the blue curve a hypothetical curve of Eq. (2) with $R_{\mathrm{b}}(2 V)$, and the red another hypothetical curve with $R_{\mathrm{b}}(3 \mathrm{~V})$. The operating condition $(P, Q)$ marked as black circle lies between $P_{\mathrm{do}}^{\mathrm{c}}(2 V)<P_{\mathrm{do}}<P_{\mathrm{do}}^{\mathrm{c}}(V)$, which results in one droplet being trapped and two droplets coalescencing. Then, by decreasing $P_{\mathrm{do}}$ to $P_{\mathrm{do}}=P^{\prime}$ (blue circle), one can make the operating condition lie in $P_{\mathrm{do}}^{\mathrm{c}}(3 V)<P_{\mathrm{do}}<P_{\mathrm{do}}^{\mathrm{c}}(2 V)$, which results in two droplets being trapped and three droplets coalescencing. The trapping and coalescence of a controlled number of droplets is demonstrated in ESI 4 experimentally.

\section{Reference}

[S1] C. Holtze et al., "Biocompatible Surfactants for Water-in-fluorocarbon Emulsions." Lab on a Chip, 2008, 8, 1632-1639. 